\title{
2017 American Brachytherapy Society's Annual Meeting Report
}

\section{Citation}

Orio, Peter F., Cathryn Yashar, Dan Petereit, Firas Mourtada, Ann Klopp, David Gaffney, and Steven Frank. 2017. “2017 American Brachytherapy Society's Annual Meeting Report.” Translational Andrology and Urology 6 (5): 1005-1013. doi:10.21037/tau.2017.09.05. http:// dx.doi.org/10.21037/tau.2017.09.05.

\section{Published Version}

doi:10.21037/tau.2017.09.05

\section{Permanent link}

http://nrs.harvard.edu/urn-3:HUL.InstRepos:34493134

\section{Terms of Use}

This article was downloaded from Harvard University's DASH repository, and is made available under the terms and conditions applicable to Other Posted Material, as set forth at http:// nrs.harvard.edu/urn-3:HUL.InstRepos:dash.current.terms-of-use\#LAA

\section{Share Your Story}

The Harvard community has made this article openly available.

Please share how this access benefits you. Submit a story.

\section{Accessibility}




\title{
2017 American Brachytherapy Society's Annual Meeting Report
}

\author{
Peter F. Orio ${ }^{1,2}$, Cathryn Yashar $^{3}$, Dan Petereit ${ }^{4}$, Firas Mourtada ${ }^{5}$, Ann Klopp ${ }^{6}$, David Gaffney ${ }^{7}$, Steven Frank ${ }^{6}$ \\ ${ }^{1}$ Harvard Medical School, Boston, Massachusetts, USA; ${ }^{2}$ Department of Radiation Oncology, Brigham and Women's Hospital/Dana-Farber Cancer \\ Institute, Boston, Massachusetts, USA; ${ }^{3}$ Department of Radiation Medicine and Applied Sciences, University of California San Diego, La Jolla, \\ California, USA; ${ }^{4}$ Rapid City Regional Cancer Care Institute, Rapid City, South Dakota, USA; ${ }^{5}$ Department of Radiation Oncology, Helen F. \\ Graham Cancer Center, Newark, Delaware, USA; ${ }^{6}$ Department of Radiation Oncology, The University of Texas MD Anderson Cancer Center, \\ Houston, Texas, USA; ${ }^{7}$ Department of Radiation Oncology, Huntsman Cancer Institute, University of Utah, Salt Lake City, Utah, USA \\ Correspondence to: Peter F. Orio. Harvard Medical School, Boston, Massachusetts, USA; Department of Radiation Oncology, Brigham and Women's \\ Hospital/Dana-Farber Cancer Institute, Boston, Massachusetts, USA. Email: Peter_Orio@dfci.harvard.edu.
}

\begin{abstract}
The American Brachytherapy Society (ABS) and its membership seeks to benefit patients by promoting the highest possible standard for brachytherapy practice, support health care professionals through the encouragement of state of the art technology and education, promote clinical and laboratory research, and advocate for the socioeconomic aspects of brachytherapy. The 2017 ABS Annual Meeting took place is Boston, Massachusetts, United States from April 20-22, 2017. The theme "The Value of Brachytherapy in Multidisciplinary Cancer Care" drew a multitude of national and international speakers to present data and debate clinical indications, advancements in practice and the value of brachytherapy. With a focus on the advancement of brachytherapy for prostate cancer and the socioeconomic benefits of brachytherapy, the globally focused program hosted 93 speakers, 506 attendees, and exhibitors from 15 countries and featured 251 abstracts for presentation and display. The ABS Annual Meeting left attendees with initial data on timely and relevant topics such as outcomes following brachytherapy for recurrent prostate cancer following external beam radiotherapy, findings of prototype algorithms capable of rapidly generating prostate brachytherapy pre-operative treatment plans and results on the socioeconomic disparities impacting the utilization of brachytherapy for common malignancies. These novel findings, among many other thoughtful and thought-provoking presentations, gave meeting attendees knowledge of the current state of brachytherapy and future directions of the specialty.
\end{abstract}

Keywords: American Brachytherapy Society; brachytherapy; prostate cancer; socioeconomic efficacy

Submitted Jul 19, 2017. Accepted for publication Aug 15, 2017.

doi: $10.21037 /$ tau. 2017.09.05

View this article at: http://dx.doi.org/10.21037/tau.2017.09.05

\section{Plenary session highlights}

The Judith Stitt Best Abstract Award recipients are selected through the ABS's meticulous scientific committee review process and the recipients' abstracts are presented in the Annual Meeting's plenary session. The 2017 Annual Meeting saw hundreds of abstract submissions, and four abstracts stood out to receive the award named for and established in the memory of Dr. Judith Stitt, who was very active in the ABS. The first recipient, Nicolae et al. presented the Evaluation of a Machine-Learning Algorithm for Treatment Planning in Prostate Low-Dose-Rate (LDR) Brachytherapy with initial findings suggesting that their prototype algorithm demonstrates the capability to generate LDR prostate brachytherapy pre-operative treatment plans (1). The authors found that their plans are comparable to the quality of plans which were simultaneously created by experts trained in brachytherapy (Figure 1) (1). The authors suggested that in looking to the future, automated planning algorithms for brachytherapy will improve uniformity of plans, reduce planning time, and decrease errors and the number of staff resources needed for brachytherapy (1). Researchers working on NRG/RTOG 0526, a prospective multicenter trial reporting on the outcomes of salvage LDR 

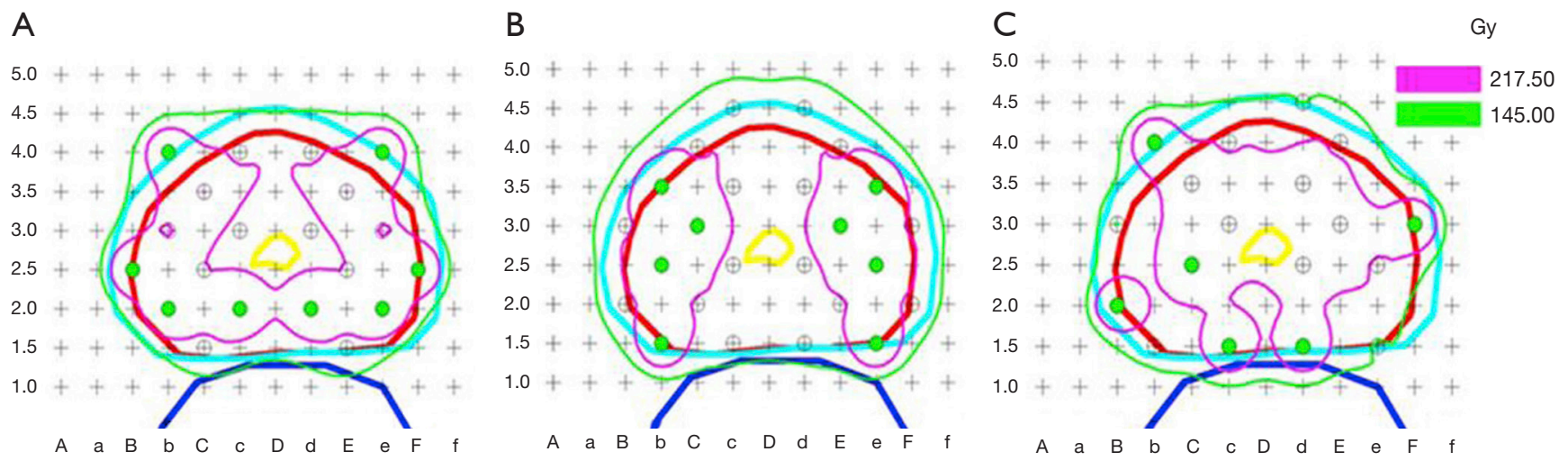

Figure 1 Demonstration of axial isodose distributions for a single patient showing (A) the ML plan, (B) the expert BT plan and (C) a generic optimization algorithm plan (1).

brachytherapy following External Beam Radiation Therapy (EBRT) received the second award and presented initial findings in A Prospective Phase II Trial of Trans-Perineal Ultra-Sound-Guided Brachytherapy for Locally Recurrent Prostate Cancer After External Beam Radiotherapy (NRG/ RTOG 0526) (2). The investigators discussed that the study's rate of late grade 3 adverse events was reported at $14 \%$, without any grade $4-5$ events and were comparable to previous study findings (2). The importance of implant dose as a predictive factor of late adverse events was discussed as well as the need for meticulous planning and technique to limit the final delivered dose (2). Clinical outcomes of this study will be reported when minimum 5 -year follow-up data is available (2).

The third award recipient, Sparks et al., presented their findings regarding long-term outcomes for patients with choroidal melanoma treated with $\mathrm{I}^{125}$ eye plaque brachytherapy (3). The group's institutional experience found that patients treated with $\mathrm{I}^{125}$ eye plaque brachytherapy had similar local control and metastases rates and had better visual preservation in comparison to those outcomes reported by Collaborative Ocular Melanoma Studies (3). And finally, in the fourth award recipient's presentation investigators discussed that the incomplete $\mathrm{PET} / \mathrm{CT}$ response rate in cervical cancer patients was documented in $17 \%$ of centrally reviewed patients following MRI based brachytherapy for cervical cancer (4). Results showed that the false positive rate of PET-IR was $74 \%$, and integration of functional MRI helped further differentiate true positives from false positives (4). Kalash et al. concluded as part of their research that the value of functional MRI requires further validation in a prospective large dataset, as this imaging modality may allow for earlier intervention and salvage treatment in those with PET-IR (4).

The Judith Stitt Best Abstract Award recipients highlight the clinical advancements of brachytherapy across many disease sites as well as the research that at this time is focusing on advanced technologies. Utilization of advanced technologies such as planning algorithms and MRI technologies will increase the precision of treatment delivery, subsequently improving outcomes and decreasing toxicities for patients in the long term.

\section{Advances in prostate brachytherapy: it's role in the treatment course and MRI integration}

Advances in prostate brachytherapy in recent years have focused on its utilization in combination with EBRT for patients with high risk disease and the utilization of MRI guided therapies for more targeted anatomical delineation and improved outcomes. Results of the study entitled Lack of Benefit Associated with External Beam Radiotherapy in Addition to Brachytherapy for Intermediate- to High-Risk Prostate Cancer suggest, when adjusting for patient-specific factors, that eligible patients with intermediate-risk or high-risk prostate cancer treated with brachytherapy might not benefit from the additional use of EBRT in their treatment course (5). The findings of this study were consistent with the recent randomized trial showing that men with relatively favorable intermediate-risk disease can be treated with brachytherapy alone (5). Studies such as these provide a framework for future prospective trials evaluating brachytherapy with ADT for patients with unfavorable-intermediate or highrisk disease, according to the researchers (5). 


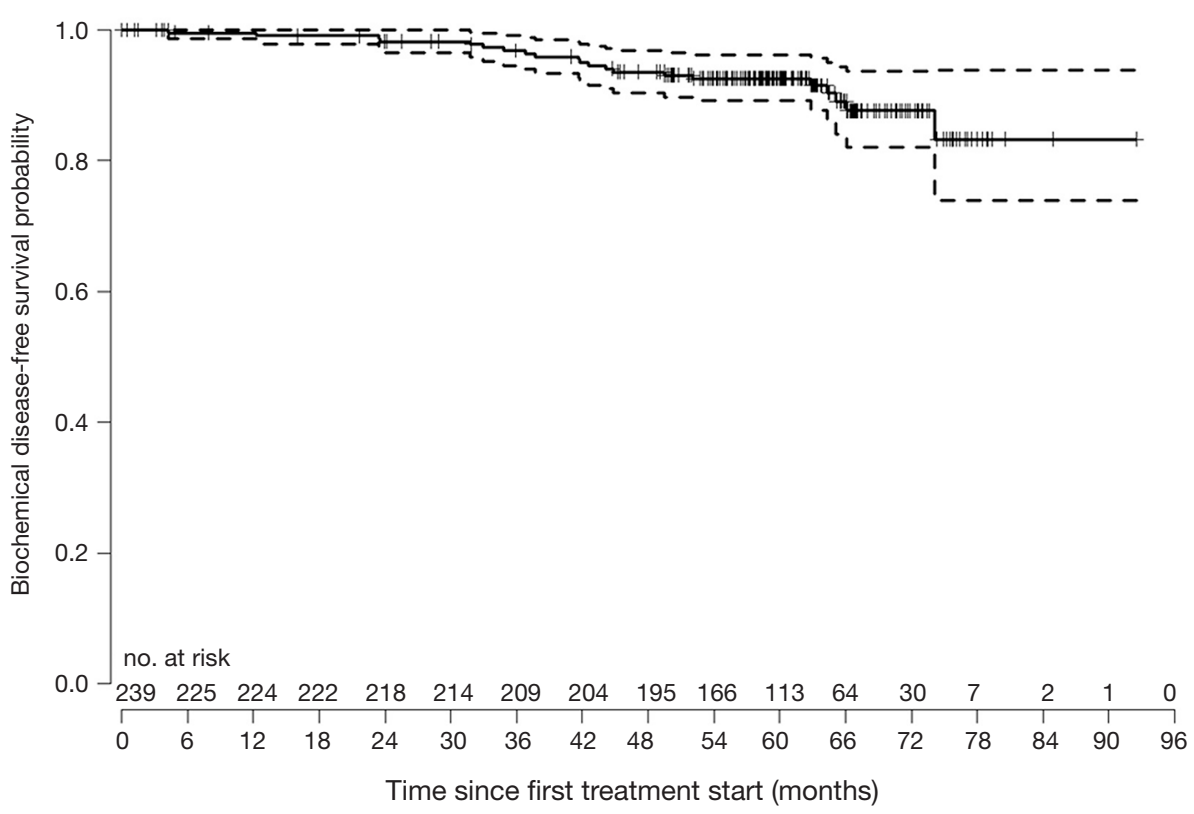

Figure 2 Probability of biochemical disease-free survival following high dose-rate (HDR) brachytherapy boost delivered as a single fraction with real-time transrectal ultrasound (TRUS) guidance (6).

The Annual Meeting also featured the work of Pearse et al., who investigated and found the use of real time transrectal ultrasound-based planning for high doserate brachytherapy boost, using a 15 Gy implant, for intermediate and high-risk prostate cancer is associated with favorable outcomes (Figure 2) (6). The group's findings are in line with the results of their previous study for intermediate risk patients (6). All et al. sought to determine that feasibility and outcomes of MRI-Based planning for high dose rate (HDR) brachytherapy for prostate cancer (7). The group's research suggested that MRI-based treatment planning is feasible for the delivery of HDR brachytherapy for prostate cancer (7). The group's plans met stringent dosimetric criteria with more accurate target and normal tissue definition (Figure 3) (7). Given these results, the group's institution has adopted MRI as their standard imaging modality for HDR brachytherapy for prostate cancer (7). Although an opportunity remains for improved efficiency in their process, the authors concluded that patient treatment time is reasonable utilizing MRI based planning (7).

Poulin et al. sought to show validation of MRI to ultrasound (US) registration for focal HDR prostate brachytherapy (8). The group concluded that deformable registration was significantly more accurate than rigid registration for brachytherapy MRI-US fusion (Figure 4) (8). Using both Hausdorff distance and TRE for common points, the study demonstrated that the deformable registration is sufficiently accurate and precise for use in focal HDR prostate brachytherapy (8). Sanders et al. argued that being able to perform accurate dosimetry using MRI images without endorectal coil is important for more widespread adoption of MRI in prostate brachytherapy (9). Their results, presented in the abstract entitled MRIBased Prostate Brachytherapy - Imaging with and without an Endorectal Coil for Post-Implant Quality Assurance, indicated that optimization in pulse sequences and scan protocols shows promise in achieving images of sufficient quality to visualize both anatomy and MRI markers adjacent to the radioactive seeds in prostate brachytherapy (Figure 5) (9). The researchers suggested that additional technical development such as higher field strength, better pulse sequences and image reconstruction algorithms, and optimized marker technologies may further improve image quality in the future (9). Martin $e t$ al. researched the utilization of automated detection of post-implant prostate brachytherapy seeds on MRI (10). The group found that automated detection is feasible, with good accuracy, enabling MRI only post-implant dosimetry (10). According to the findings, the utilization of this reduces the number of 


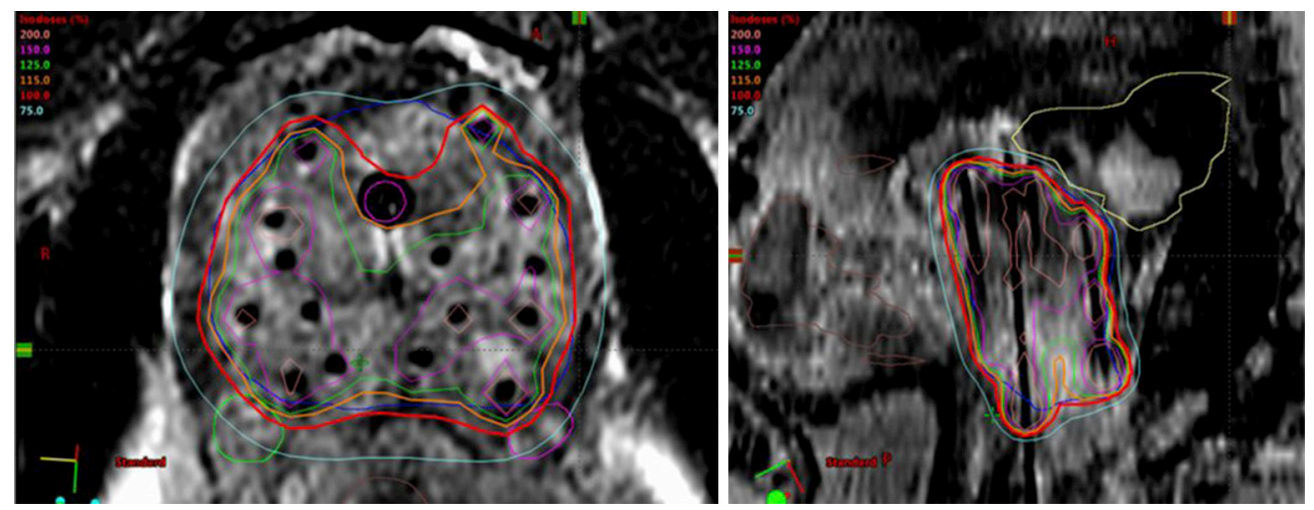

Figure 3 Pre-treatment DVH goals to the target were: $\mathrm{D}_{90} \geq 95 \%, \mathrm{~V}_{90} \geq 95 \%, \mathrm{~V}_{100} \geq 90 \%, \mathrm{~V}_{150} \leq 30 \%, \mathrm{~V}_{200} \leq 15 \%$. DVH goals to normal structures were: urethra $\mathrm{D}_{.01 \mathrm{cc}} \leq 115 \%$, bladder D1cc $\leq 75 \%$, rectum $\mathrm{D}_{1 \mathrm{cc}} \leq 75 \%$, neurovascular bundle $\mathrm{D}_{0.1 \mathrm{cc}} \leq 100 \%$, penile bulb $\mathrm{D}_{1 \mathrm{cc}} \leq 100 \%(7)$.

brachytherapy seeds which need to be identified manually and suggested that their continued research will work to refine the algorithm to focus on reduced computational time and improved seed detection in the future (10).

The utilization of MRI technologies in the planning and delivery of prostate brachytherapy is a technique which has grown in investigational and clinical use over the past decade. Although changes in clinical practice and training are required to utilize MRI for planning and delivery of prostate brachytherapy research reported at the Annual Meeting shows promise that its utilization can decrease toxicity to surrounding tissues and organs at risk and that it has the ability to more accurately delineate disease and surrounding anatomy for more accurate dose distribution. The utilization of MRI has the potential to better delineate both prostate anatomy and disease, increasing the rate of positive clinical outcomes and decreasing side effects for patients.

\section{Advances in prostate brachytherapy: outcomes and toxicities}

In addition to research focused on MRI technologies, significant research in brachytherapy has focused on improved outcomes and reduced toxicities. The study completed and presented by Yorozu et al., Predictive Factors of Long-Term Rectal Toxicity Following I-125 Prostate Brachytherapy with or without External Beam Radiotherapy, found that prostate brachytherapy utilizing I-125 seeds, especially when used as monotherapy, is well tolerated (11). The researchers concluded that rectal dosimetry is relevant to long-term rectal toxicity following brachytherapy in both the monotherapy and boost setting following EBRT (11).
The group's results showed with rectal dose constraints that are both proper and achievable, EBRT yielded less toxicity when used in combination with brachytherapy (11).

Hegde et al. reported on a study of men with highrisk prostate cancer treated with EBRT plus an HDR brachytherapy boost and found that MRI evidence of seminal vesicle invasion predicted for biochemical failure and that pre-treatment clinical and biopsy variables were not predictive, as commonly believed (12). The group concluded that pre-treatment multiparametric MRI can be useful for identifying men who are at higher risk for biochemical failure following a EBRT and HDR brachytherapy boost treatment (12). Vigneault et al. researched the different HDR brachytherapy boost treatment courses at their individual institution and found that an increase in BED is correlated with an improvement of biochemical control, as defined in their research a nPSA $<0.4 \mathrm{ng} / \mathrm{mL}$ (13). The group found that improvement in biochemical control, however, is also associated with slight, but clinically acceptable, increase in urinary toxicity (13). Morris et al. found that using a Surgical PSA-Threshold $(>0.2 \mathrm{ng} / \mathrm{mL})$ of biochemical failure in the ASCENDE-RT population greatly increased the number and percentage of biochemical relapse events for the subset of trial patients that received an external beam boost (14). However, the group found that the application of this threshold had little effect on the subset of patients who received a brachytherapy boost (14).

Morton et al. investigated the use of one versus two fractions of Prostate HDR monotherapy and presented short term follow up data which suggested that higher PSA values and higher local recurrence rates were found in the 

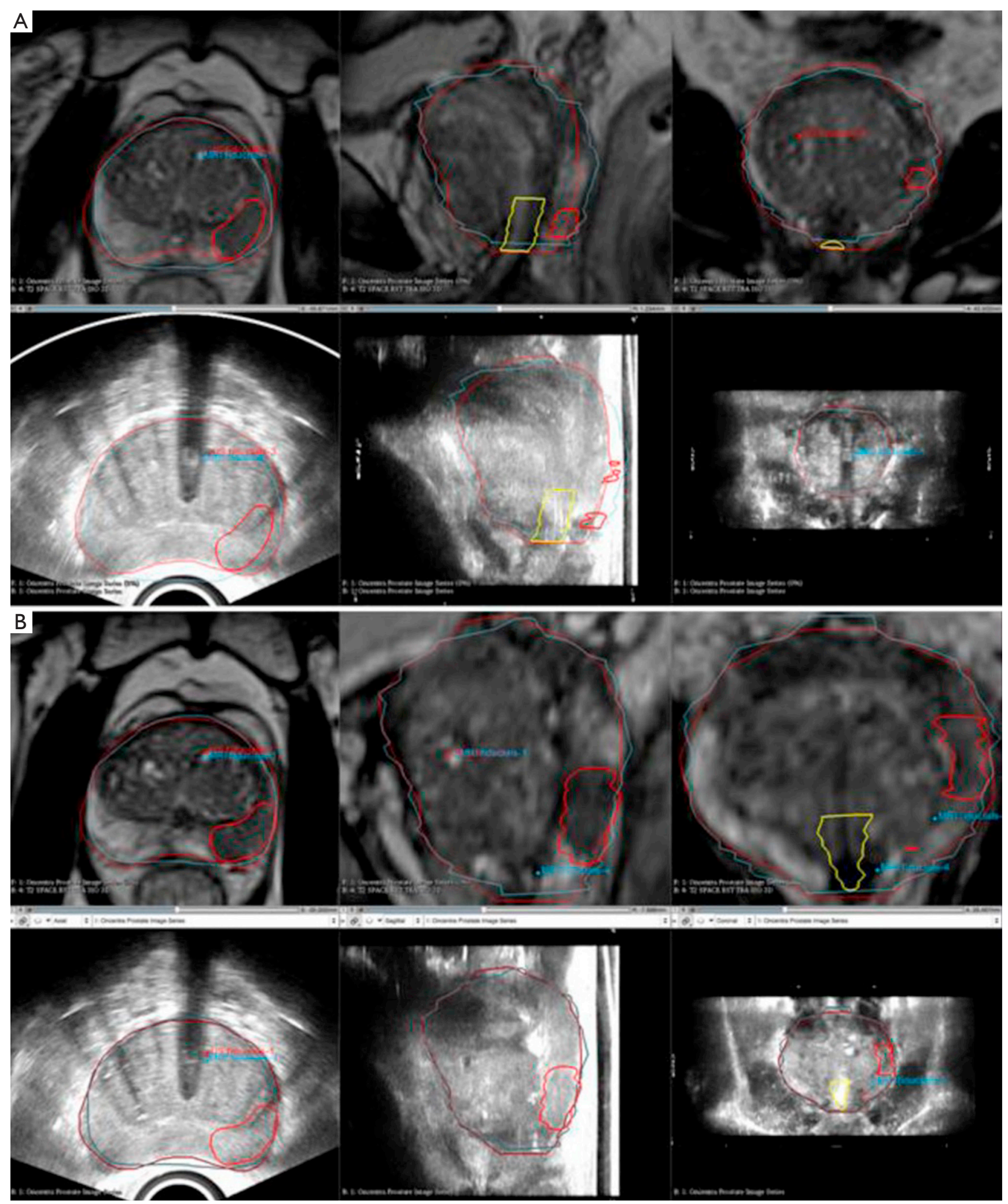

Figure 4 A representative registration between MRI (blue) and US (red) contours for (A) rigid and (B) deformable registration (8). 


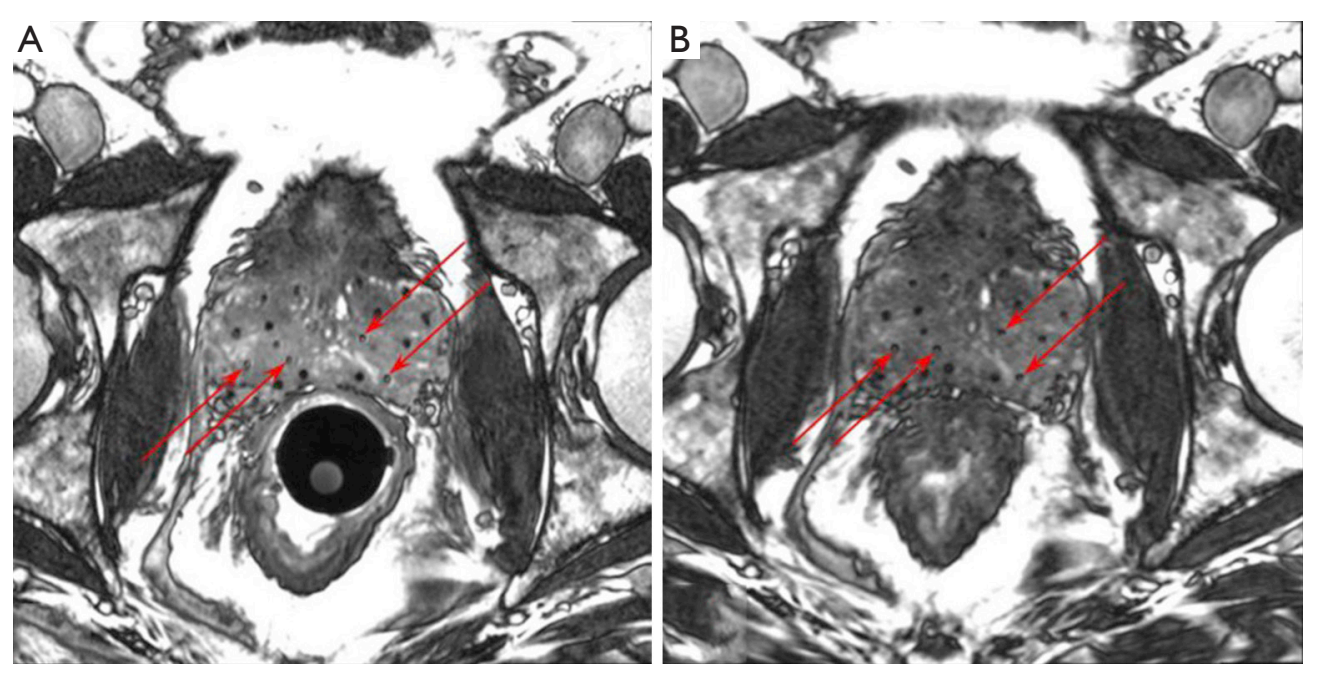

Figure 5 Comparison of the MR images acquired for the same patient with (A) and without (B) the ERC (9).

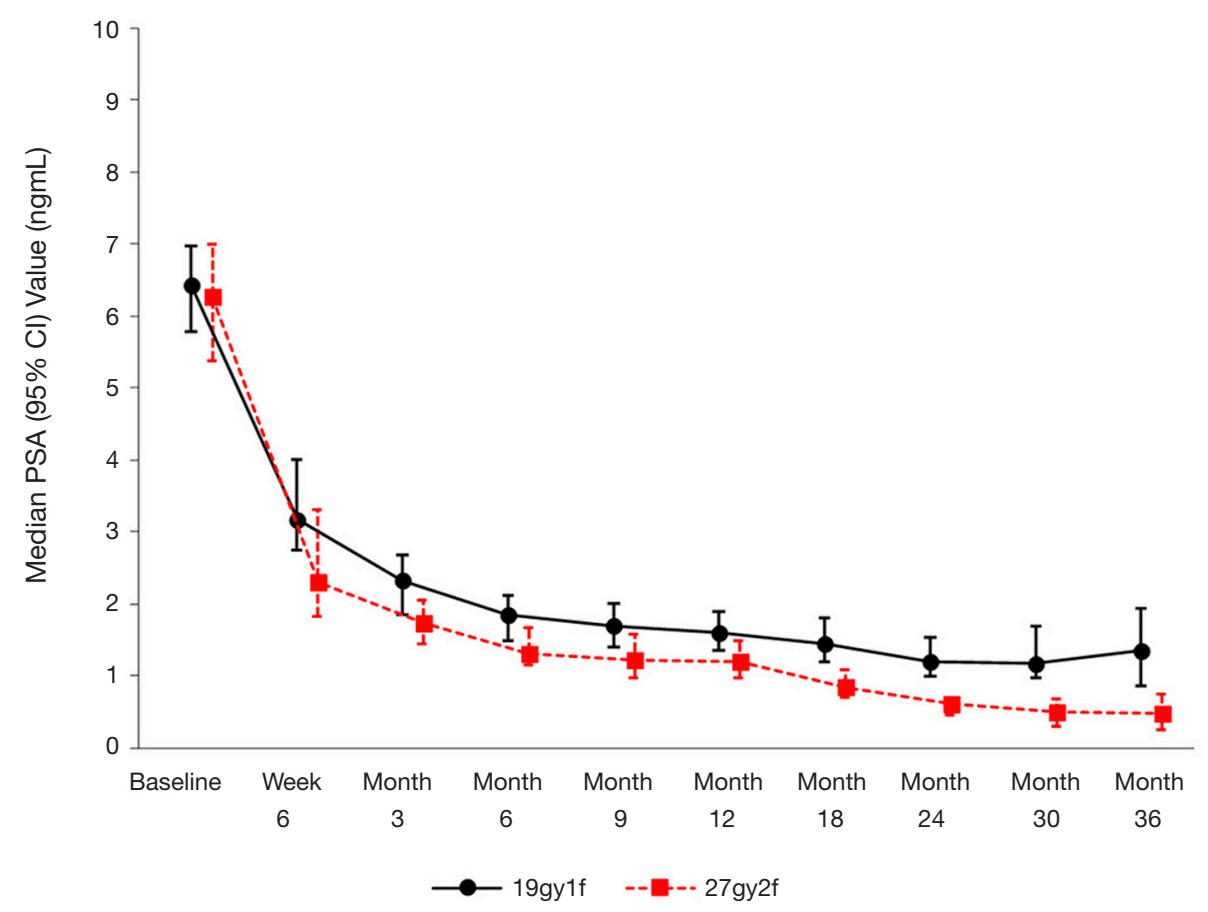

Figure 6 Median PSA values for high dose-rate (HDR) brachytherapy monotherapy as either 19 Gy $\times 1$ or 13.5 Gy $\times 2$ in early stage prostate cancer (15).

single fraction arm (15). The group suggested that $19 \mathrm{~Gy}$ does not appear to be biologically equivalent to $27 \mathrm{~Gy}$ in 2 fractions and suggested that a single 19 Gy fraction should be used with caution as monotherapy (Figure 6) (15).

Researchers who presented data on prostate brachytherapy treatment, outcome and toxicity findings at the
2017 Annual Meeting relayed the importance of dosimetry in predicting long-term toxicity and BED calculations following brachytherapy as well as predictive factors for outcomes and the importance of defining failure and relapse thresholds. Prostate brachytherapy researchers continue to work to improve the efficacy, outcomes and toxicities of this 
Table 1 Changes on the quality of life after I-125 seed implantation in patients with advanced malignant tumor (20)

\begin{tabular}{lcccc}
\hline Items & $\begin{array}{c}\text { Before the seeds } \\
\text { implantation in 24 h }\end{array}$ & $\begin{array}{c}\text { After the seeds } \\
\text { implantation in 24 h }\end{array}$ & $\begin{array}{c}\text { 1 month after the seeds } \\
\text { implantation }\end{array}$ & $\begin{array}{c}3 \text { months after the } \\
\text { seeds implantation }\end{array}$ \\
\hline Total score in EORTCQLQ-C30 & $82.63 \pm 10.419$ & $86.38 \pm 20.853$ & $96.75 \pm 21.029$ & $89.86 \pm 22.645$ \\
Total health performance in 1 week & $4.10 \pm 1.708$ & $4.26 \pm 1.624$ & $4.8 \pm 1.389$ & $4.11 \pm 1.491$ \\
Total quality of life in 1 week & $4.14 \pm 1.719$ & $4.26 \pm 1.712$ & $4.83 \pm 1.403$ & $3.94 \pm 1.893$ \\
\hline
\end{tabular}

treatment modality to better treat patients with this disease.

\section{Brachytherapy's socioeconomic efficacy highlights}

Socioeconomic factors are crucial considerations in the implementation, utilization and future of brachytherapy across the globe. In recent years, the research on socioeconomic factors impacting the utilization of brachytherapy have focused on program implementation considerations as well as the socioeconomic status and demographics of patient populations and the country of implementation.

HDR brachytherapy for cervical cancer has been successfully established in Nicaragua, a developing country with limited resources and a high burden of disease per the presentation by Nurkic et al. (16). The center achieved a program through a dedicated HDR room apart from established EBRT rooms and through implementation of a multi-phase treatment plan and delivery workflow (16). Although the group reported that challenges remain in regards to patient access, dated technology and software, as well as equipment maintenance, they are comfortable reporting on the successful implementation of the program in the low-middle income country (16). This successful example of implementation of an HDR program in a resource-limited environment could serve to guide other countries in similar circumstances in the future (16). The authors of the abstract entitled "Starting a Prostate HDR Program in a Young Cancer Center - 1st Year Experience" reported that the implementation of a new HDR prostate brachytherapy program should be approached as a continual improvement process (17). The study group found that the planning of details and communication between all members of the multidisciplinary team is imperative for successful protocol implementation (17). Using an iterative validation process approach was fundamental in improving the efficacy and made the group able to improve both quality and efficiency in the process according to researchers (17).

The authors of "Social and Racial Divides in the Utilization of Brachytherapy for Common Malignancies" utilized a large, population-based analysis to show that it is evident that disparities in care utilizing brachytherapy exist because of both social and racial factors (18). The researchers suggested that these gaps need to be further examined and remedied to provide standard of care therapy to all patients (18).

Vega et al. researched the factors which are associated with a willingness of organizations to invest in a HDR program utilizing Co-60 and found that decision makers in $\mathrm{RT}$ equipment acquisition had low willingness to invest in shielding should an HDR source more costeffective than Ir-192 become available (19). Other factors influential included ABS membership and having attended a national conference for brachytherapy, and centers with a higher number of attending physicians was associated with higher willingness to invest (19). The group concluded that given the results, larger centers with greater exposure to brachytherapy may be more likely to adopt a new HDR source in the future (19). The group argued that centers currently shielded for Co-60 may pursue the utilization of this source, but that research appears to show that the market is unfavorable for Co-60 in centers that would require shielding expansion for adoption (19).

The abstract "Quality of Life After I-125 Seeds Implantation in Patients with Advanced Malignant Tumor", presented by Wang et al. found that seed implantation can improve the quality of life in patients with advanced malignant tumors (20). Analysis showed that 3 months after seed implantation, patients' quality of life improved, but after 3 months, the patients' quality of life declined and may be influenced by the tumor progression (Table 1) (20). Bajaj et al. discussed the successful reduction of treatment planning duration by $40 \%$ through optimization of clinical throughput, resulting in their HDR program having similar treatment time duration as compared to their LDR seed implant procedures (21). 
The utilization of brachytherapy is influenced by societal and economic factors as evidence from the presentations of researchers at the Annual Meeting. Although hurdles exist, such as the cost of implementation and training needed to run brachytherapy programs, it remains a clinically and cost-effective treatment option for patients with many disease site diagnoses. With economic and societal considerations effecting healthcare across the globe, the ABS and its membership remain dedicated to ensuring that researchers, physicians and hospital administrators are aware of the economic and patient related outcome data that encourage the implementation and utilization of a brachytherapy program within radiation oncology departments.

\section{Conclusions}

"The Value of Brachytherapy in Multidisciplinary Cancer Care" was evident at ABS' 2017 Annual Meeting through the presentation of hundreds of abstracts that focused on the ways in which brachytherapy can positively impact clinical outcomes, overall survival and patient reported outcomes. Additionally, the value of brachytherapy was evident in the socioeconomic presentations from individuals across the globe. The ABS Annual Meeting featured novel findings of the development of MRI inclusion, algorithm and predictive programming progression for brachytherapy and the range of cancerous diseases which can benefit from the inclusion of brachytherapy during the course of care. Since 1978 the ABS and its membership have provided insight, rationale and research to clinicians on brachytherapy's efficacy and they will continue to do so at the 2018 Annual Meeting, scheduled to be held June 07-09, 2018 in San Francisco, California.

\section{Acknowledgements}

None.

\section{Footnote}

Conflicts of Interest: The authors have no conflicts of interest to declare.

\section{References}

1. Nicolae A, Morton G, Chung H, et al. Evaluation of a Machine-Learning Algorithm for Treatment Planning in
Prostate Low-Dose-Rate Brachytherapy. Paper Presented at: American Brachytherapy Society Annual Meeting. Boston, Massachusetts, United States. April, 2017.

2. Crook J, Zhang P, Pisansky T, et al. A Prospective Phase II Trial of Trans-Perineal Ultra-Sound-Guided Brachytherapy for Locally Recurrent Prostate Cancer After External Beam Radiotherapy (NRG/RTOG 0526). Paper Presented at: American Brachytherapy Society Annual Meeting. April, 2017, Boston, Massachusetts, United States. April, 2017.

3. Sparks I, Wirostko W, Rownd J, et al. Long Term Outcomes of I125 Eye Plaque Brachytherapy in Patients with Choroidal Melanoma. Paper Presented at: American Brachytherapy Society Annual Meeting. Boston, Massachusetts, United States. April, 2017.

4. Kalash R, Rangaswamy B, Glaser S, et al. The Use of Functional MRI in Cervical Cancer Patients with Incomplete Response on PET/CT Following MRI Guided High-Dose Rate Brachytherapy. Paper Presented at: American Brachytherapy Society Annual Meeting. Boston, Massachusetts, United States. April, 2017.

5. Muralidhar V, Orio PF, III, Nguyen PL, et al. Lack of Benefit Associated with External Beam Radiotherapy in Addition to Brachytherapy for Intermediate- to HighRisk Prostate Cancer. Paper Presented at: American Brachytherapy Society Annual Meeting. Boston, Massachusetts, United States. April, 2017.

6. Pearse J, Song W, Loblaw A, et al. Real-Time Transrectal Ultrasound-Based Planning for High DoseRate Brachytherapy Boost in Intermediate and High Risk Prostate Cancer. Paper Presented at: American Brachytherapy Society Annual Meeting. Boston, Massachusetts, United States. April, 2017.

7. All S, Keller A, Warner C, et al. Feasibility and Dosimetric Outcomes of MRI-Based Planning for Delivery of HighDose-Rate Brachytherapy for Prostate Cancer. Paper Presented at: American Brachytherapy Society Annual Meeting. Boston, Massachusetts, United States. April, 2017.

8. Poulin E, Boudam K, Pinter C, et al. Validation of MRI to US Registration for Focal HDR Prostate Brachytherapy. Paper Presented at: American Brachytherapy Society Annual Meeting. Boston, Massachusetts, United States. April, 2017.

9. Sanders J, Frank S, Bathala T, et al. MRI-Based Prostate Brachytherapy - Imaging with and without an Endorectal Coil for Post-Implant Quality Assurance. Paper Presented at: American Brachytherapy Society Annual Meeting. Boston, Massachusetts, United States. April, 2017. 
10. Martin GV, Ma J, Kudchadker RJ, et al. Automated Prostate Brachytherapy Seed Detection on Post-Implant MRI Using Novel Martin Algorithm. Paper Presented at: American Brachytherapy Society Annual Meeting. Boston, Massachusetts, United States. April, 2017.

11. Yorozu A, Sutani S, Sunaguchi A, et al. Predictive Factors of Long-Term Rectal Toxicity Following I-125 Prostate Brachytherapy with or without External Beam Radiotherapy. Paper Presented at: American Brachytherapy Society Annual Meeting. Boston, Massachusetts, United States. April, 2017.

12. Hegde JV, Demanes DJ, Veruttipong D, et al. PreTreatment MRI Staging Predicts for Biochemical Failure in High-Risk Prostate Cancer Treated with Combination High-Dose-Rate Brachytherapy and External Beam Radiotherapy. Paper Presented at: American Brachytherapy Society Annual Meeting. Boston, Massachusetts, United States. April, 2017.

13. Vigneault E, Mbodji K, Magnan S, et al. Effect of Different Hypofractionated Regimens Combination on Clinical Outcomes in Prostate Cancer Patients Treated with High Dose-Rate Brachytherapy Boost. Paper Presented at: American Brachytherapy Society Annual Meeting. Boston, Massachusetts, United States. April, 2017.

14. Morris WJ, Keyes M, Pickles T. Using a Surgical PSAThreshold (> $0.2 \mathrm{ng} / \mathrm{mL}$ ) to Define Biochemical Failure in the ASCENDE-RT Phase 3 Trial. Paper Presented at: American Brachytherapy Society Annual Meeting. Boston, Massachusetts, United States. April, 2017.

15. Morton G, Chung H, McGuffin M, et al. Prostate HDR Monotherapy: Initial Efficacy Results from a Randomized Trial of One versus Two Fractions. Paper Presented at:

Cite this article as: Orio PF, Yashar C, Petereit D, Mourtada F, Klopp A, Gaffney D, Frank S. 2017 American Brachytherapy Society's Annual Meeting Report. Transl Androl Urol 2017;6(5):1005-1013. doi: 10.21037/tau.2017.09.05
American Brachytherapy Society Annual Meeting. Boston, Massachusetts, United States. April, 2017.

16. Nurkic S, Ocampo A, Gadea M, et al. The Successful Implementation of High Dose Rate 192-Ir Brachytherapy for Cervix Cancer in a Low-Middle Income Country. Paper Presented at: American Brachytherapy Society Annual Meeting. Boston, Massachusetts, United States. April, 2017.

17. Lauzon A, Piché É, Roy S, et al. Starting a Prostate HDR Program in a Young Cancer Center - 1st Year Experience. Paper Presented at: American Brachytherapy Society Annual Meeting. Boston, Massachusetts, United States. April, 2017.

18. Horn ZD, Balassubramani GK, Sukumvanich P, et al. Social and Racial Divides in the Utilization of Brachytherapy for Common Malignancies. Paper Presented at: American Brachytherapy Society Annual Meeting. Boston, Massachusetts, United States. April, 2017.

19. Vega RM, Talcott W, Ishaq O, et al. Factors Associated with Willingness to Invest in a New HDR Isotope. Paper Presented at: American Brachytherapy Society Annual Meeting. Boston, Massachusetts, United States. April, 2017.

20. Wang PP, Zhang M, Ji Z, et al. Quality of Life After I-125 Seeds Implantation in Patients with Advanced Malignant Tumor. Paper Presented at: American Brachytherapy Society Annual Meeting. Boston, Massachusetts, United States. April, 2017.

21. Bajaj A, Korpics M, Martin B, et al. Reducing Prostate High Dose Rate Brachytherapy Treatment Planning Duration Through Targeted Interventions. Paper Presented at: American Brachytherapy Society Annual Meeting. Boston, Massachusetts, United States. April, 2017. 